\title{
A unified approach to fractal Hilbert-type inequalities
}

\author{
Tserendorj Batbold ${ }^{1 *}$, Mario Krnić ${ }^{2}$ and Predrag Vuković ${ }^{3}$
}

"Correspondence:

tsbatbold@hotmail.com

'Department of Mathematics,

National University of Mongolia,

Ulaanbaatar, Mongolia

Full list of author information is

available at the end of the article

\begin{abstract}
In the present study we provide a unified treatment of fractal Hilbert-type inequalities. Our main result is a pair of equivalent fractal Hilbert-type inequalities including a general kernel and weight functions. A particular emphasis is devoted to a class of homogeneous kernels. In addition, we impose appropriate conditions for which the constants appearing on the right-hand sides of the established inequalities are the best possible. As an application, our results are compared with some previously known ones from the literature.
\end{abstract}

MSC: 26D15

Keywords: Hilbert inequality; Conjugate parameters; Local fractional integral; Homogeneous function; The best possible constant

\section{Introduction}

The celebrated Hilbert inequality (see [8]) in its integral form asserts that

$$
\int_{0}^{\infty} \int_{0}^{\infty} \frac{f(x) g(y)}{x+y} d x d y \leq \frac{\pi}{\sin \frac{\pi}{p}}\left[\int_{0}^{\infty} f^{p}(x) d x\right]^{\frac{1}{p}}\left[\int_{0}^{\infty} g^{q}(y) d y\right]^{\frac{1}{q}},
$$

where $f, g:(0, \infty) \rightarrow \mathbb{R}$ are non-negative integrable functions and $p, q$ is a pair of nonnegative conjugate exponents, i.e., $\frac{1}{p}+\frac{1}{q}=1, p>1$. In addition, the constant $\frac{\pi}{\sin \frac{\pi}{p}}$ is the best possible in the sense that it cannot be replaced by a smaller positive constant so that the inequality remains valid. Hardy et al. [8], noticed that one can assign to (1) its equivalent form

$$
\int_{0}^{\infty}\left[\int_{0}^{\infty} \frac{f(x)}{x+y} d x\right]^{p} d y \leq\left(\frac{\pi}{\sin \frac{\pi}{p}}\right)^{p} \int_{0}^{\infty} f^{p}(x) d x
$$

in the sense that (1) implies (2) and vice versa. During decades, Hilbert-type inequalities (1) and (2) have been extensively studied by numerous authors. A rich variety of extensions included inequalities with more general kernels, weight functions, and integration domains, as well as refinements of initial Hilbert-type inequalities (1) and (2). It is important to point out that these inequalities are still of interest to numerous authors. For an initial development of the Hilbert-type inequalities, the reader is referred to [8], while some recent results are collected in monographs [3] and [10].

(c) The Author(s) 2019. This article is distributed under the terms of the Creative Commons Attribution 4.0 International License (http://creativecommons.org/licenses/by/4.0/), which permits unrestricted use, distribution, and reproduction in any medium, provided you give appropriate credit to the original author(s) and the source, provide a link to the Creative Commons license, and indicate if changes were made. 
Nowadays, an interesting topic in connection to classical inequalities is their extension on certain fractal spaces via the local fractional calculus. The local fractional calculus is primarily utilized to handle various non-differentiable problems that appear in complex systems of the real-world phenomena. In particular, the non-differentiability occurring in science and engineering has been modeled by the local fractional ordinary or partial differential equations.

On the other hand, local fractional calculus is also an important tool in pure mathematics. Recently, by virtue of the local fractional calculus, a whole series of classical real inequalities have been extended to hold on certain fractal spaces. For the reader's convenience, denote by ${ }_{a} I_{b}^{\alpha} f(x)$ and ${ }_{a} I_{b}^{\alpha}\left[I_{b}^{\alpha} h(x, y)\right]$ local fractional integrals

$$
{ }_{a} I_{b}^{\alpha} f(x)=\frac{1}{\Gamma(1+\alpha)} \int_{a}^{b} f(x)(d x)^{\alpha}
$$

and

$$
{ }_{a} I_{b}^{\alpha}\left[I_{b}^{\alpha} h(x, y)\right]=\frac{1}{\Gamma^{2}(1+\alpha)} \int_{a}^{b} \int_{a}^{b} h(x, y)(d x)^{\alpha}(d y)^{\alpha}
$$

where $0<\alpha \leq 1$ and where $\Gamma$ stands for a usual gamma function defined by $\Gamma(a)=$ $\int_{0}^{\infty} t^{a-1} e^{-t} d t, a>0$. Further, let $C_{\alpha}(a, b)$ stand for a set of local fractional continuous functions on the interval $(a, b)$.

Recently, Liu and Sun [13] established a pair of equivalent fractal Hilbert-type inequalities expressed in terms of the above fractional integrals. Namely, they showed that if $\frac{1}{p}+\frac{1}{q}=1, p>1,0<\alpha \leq 1$, and if $f, g \in C_{\alpha}(a, b)$ are non-negative functions, then the following inequalities hold:

$$
\begin{aligned}
& { }_{0} I_{\infty}^{\alpha}\left[{ }_{0} I_{\infty}^{\alpha} \frac{f(x) g(y)}{\max \left\{x^{\alpha}, y^{\alpha}\right\}}\right] \\
& \quad \leq \eta(\alpha)\left[{ }_{0} I_{\infty}^{\alpha}\left(x^{\frac{\alpha}{2}(p-2)} f^{p}(x)\right)\right]^{\frac{1}{p}}\left[{ }_{0} I_{\infty}^{\alpha}\left(y^{\frac{\alpha}{2}(q-2)} g^{q}(y)\right)\right]^{\frac{1}{q}}
\end{aligned}
$$

and

$$
{ }_{0} I_{\infty}^{\alpha}\left[y^{\frac{\alpha(2-q)}{2(q-1)}}\left[{ }_{0} I_{\infty}^{\alpha} \frac{f(x)}{\max \left\{x^{\alpha}, y^{\alpha}\right\}}\right]^{p}\right]<\eta^{p}(\alpha)_{0} I_{\infty}^{\alpha}\left(x^{\frac{\alpha}{2}(p-2)} f^{p}(x)\right)
$$

where $\eta(\alpha)=\frac{2^{\alpha+1}}{\Gamma(1+\alpha)}$ and provided that the integrals on the right-hand sides of (3) and (4) are convergent. In addition, it has been also shown that the constants $\eta(\alpha)$ and $\eta^{p}(\alpha)$ are the best possible.

For some related extensions of classical inequalities to fractal spaces the reader is referred to recent papers $[4-6,9,11-13,16,17]$. In addition, for some recent results closely connected to this topic the reader is referred to $[1,2,7,15]$ and the references therein.

The main objective of the present paper is a unified treatment of fractal Hilbert-type inequalities. In other words, we will establish a pair of fractal Hilbert-type inequalities with a general kernel and general weight functions that cover the above presented Hilbert-type inequalities. The paper is divided into four sections as follows: After this introductory part, in Sect. 2 we give a brief overview of basic definitions and properties of the local 
fractional calculus that will be the main tools in establishing our results. In Sect. 3, we derive our main result, i.e., a pair of equivalent fractal Hilbert-type inequalities with a general kernel and weight functions. A particular emphasis is devoted to a class of homogeneous kernels. In addition, we impose conditions for which the constants appearing on the right-hand sides of the corresponding Hilbert-type inequalities are the best possible. As an application, in Sect. 4 we discuss some particular choices of homogeneous kernels and power weight functions. In such a way, we show that the fractal Hilbert-type inequalities presented in this introduction are consequences of our general results.

\section{Preliminaries on local fractional calculus}

For the reader's convenience, in this section we give a brief overview of the local fractional calculus. More precisely, we give basic definitions and properties of the local fractional derivative and integral developed in [19] (see also [18]).

Let $\mathbb{R}^{\alpha}, 0<\alpha \leq 1$, be an $\alpha$-type fractal set of real line numbers. For $a^{\alpha}, b^{\alpha} \in \mathbb{R}^{\alpha}$, we define addition and multiplication by

$$
a^{\alpha}+b^{\alpha}:=(a+b)^{\alpha}, \quad a^{\alpha} \cdot b^{\alpha}=a^{\alpha} b^{\alpha}:=(a b)^{\alpha} .
$$

With these two binary operations, $\mathbb{R}^{\alpha}$ becomes a field with an additive identity $0^{\alpha}$ and a multiplicative identity $1^{\alpha}$.

The starting point in introducing the local fractional calculus on $\mathbb{R}^{\alpha}$ is the concept of the local fractional continuity. A non-differentiable function $f: \mathbb{R} \rightarrow \mathbb{R}^{\alpha}$ is said to be local fractional continuous at $x_{0}$ if, for any $\varepsilon>0$, there exists $\delta>0$ such that $\left|x-x_{0}\right|<\delta$ implies that

$$
\left|f(x)-f\left(x_{0}\right)\right|<\varepsilon^{\alpha} .
$$

The set of local fractional continuous functions on interval $I$ is denoted by $C_{\alpha}(I)$.

The local fractional derivative of $f$ of order $\alpha$ at $x=x_{0}$ is defined by

$$
f^{(\alpha)}\left(x_{0}\right)=\left.\frac{d^{\alpha} f(x)}{d x^{\alpha}}\right|_{x=x_{0}}=\lim _{x \rightarrow x_{0}} \frac{\Gamma(1+\alpha)\left(f(x)-f\left(x_{0}\right)\right)}{\left(x-x_{0}\right)^{\alpha}},
$$

where $\Gamma$ stands for a usual gamma function. Now, let $f^{(\alpha)}(x)=D_{x}^{\alpha} f(x)$. If there exists $f^{(k+1) \alpha}(x)=\overbrace{D_{x}^{\alpha} \cdots D_{x}^{\alpha}}^{k+1} f(x)$ for every $x \in I$, then we denote $f \in D_{(k+1) \alpha}(I)$, where $k=0,1,2, \ldots$

The local fractional integral is defined for a class of local fractional continuous functions. Let $f \in C_{\alpha}[a, b]$ and let $P=\left\{t_{0}, t_{1}, \ldots, t_{N}\right\}, N \in \mathbb{N}$, be a partition of interval $[a, b]$ such that $a=t_{0}<t_{1}<\cdots<t_{N-1}<t_{N}=b$. Further, for this partition $P$, let $\Delta t_{j}=t_{j+1}-t_{j}, j=0, \ldots, N-1$, and $\Delta t=\max \left\{\Delta t_{1}, \Delta t_{2}, \ldots, \Delta t_{N-1}\right\}$. Then the local fractional integral of $f$ on the interval $[a, b]$ of order $\alpha$ (denoted by $\left.{ }_{a} I_{b}^{\alpha} f(x)\right)$ is defined by

$$
{ }_{a} I_{b}^{\alpha} f(x)=\frac{1}{\Gamma(1+\alpha)} \int_{a}^{b} f(t)(d t)^{\alpha}=\frac{1}{\Gamma(1+\alpha)} \lim _{\Delta t \rightarrow 0} \sum_{j=0}^{N-1} f\left(t_{j}\right)\left(\Delta t_{j}\right)^{\alpha} .
$$

The above definition implies that ${ }_{a} I_{b}^{\alpha} f(x)=0$ if $a=b$ and ${ }_{a} I_{b}^{\alpha} f(x)={ }_{b} I_{a}^{\alpha} f(x)$ if $a<b$. 
Similar to the Riemann integral, we have the following analogue of the Newton-Leibnitz formula on the fractal space. Namely, if $f=g^{(\alpha)} \in C_{\alpha}[a, b]$, then

$$
{ }_{a} I_{b}^{\alpha} f(x)=g(b)-g(a)
$$

In particular, if $f(x)=x^{k \alpha}, k \in \mathbb{R}$, then

$$
\frac{1}{\Gamma(1+\alpha)} \int_{a}^{b} x^{k \alpha}(d x)^{\alpha}=\frac{\Gamma(1+k \alpha)}{\Gamma(1+(k+1) \alpha)}\left(b^{(k+1) \alpha}-a^{(k+1) \alpha}\right) .
$$

In order to conclude our discussion regarding fractional integrals, we give a variant of the change of variables theorem in the present setting. Namely, if $g \in D_{\alpha}[a, b]$ and $(f \circ g) \in$ $C_{\alpha}[g(a), g(b)]$, then the following relation holds:

$$
{ }_{a} I_{b}^{\alpha}(f \circ g)(s)\left[g^{\prime}(s)\right]^{\alpha}={ }_{g(a)} I_{g(b)}^{\alpha} f(x) .
$$

It should be noticed here that if $\alpha=1$, then the local fractional calculus reduces to the classical real calculus. For more details about the above presented concept of fractional differentiability and integrability, the reader is referred to [19] and the references therein.

The crucial step in establishing Hilbert-type inequalities is the well-known Hölder inequality. A fractal version of the Hölder inequality asserts that if $\frac{1}{p}+\frac{1}{q}=1, p>1$, then the inequality

$$
\begin{aligned}
\frac{1}{\Gamma(1+\alpha)} \int_{a}^{b} f(x) g(x)(d x)^{\alpha} \leq & {\left[\frac{1}{\Gamma(1+\alpha)} \int_{a}^{b} f^{p}(x)(d x)^{\alpha}\right]^{\frac{1}{p}} } \\
& \times\left[\frac{1}{\Gamma(1+\alpha)} \int_{a}^{b} g^{q}(x)(d x)^{\alpha}\right]^{\frac{1}{q}}
\end{aligned}
$$

holds for all $f, g \in C_{\alpha}(a, b)$. However, we will utilize a two-variable version of the fractal Hölder inequality which claims that

$$
\begin{aligned}
& \frac{1}{\Gamma^{2}(1+\alpha)} \iint_{S^{(\beta)}} h(x, y) F(x, y) G(x, y)(d x)^{\alpha}(d y)^{\alpha} \\
& \leq\left[\frac{1}{\Gamma^{2}(1+\alpha)} \iint_{S^{(\beta)}} h(x, y) F^{p}(x, y)(d x)^{\alpha}(d y)^{\alpha}\right]^{\frac{1}{p}} \\
& \quad \times\left[\frac{1}{\Gamma^{2}(1+\alpha)} \iint_{S^{(\beta)}} h(x, y) G^{q}(x, y)(d x)^{\alpha}(d y)^{\alpha}\right]^{\frac{1}{q}}
\end{aligned}
$$

holds for all $F, G, h \in C_{\alpha}\left(S^{(\beta)}\right)$, where $S^{(\beta)}$ is a fractal surface. For the proofs of the above inequalities, the reader is also referred to [19].

Finally, to conclude this section we give a definition of a fractal beta function. Recall that the usual beta function is defined by $B(a, b)=\int_{0}^{1} t^{a-1}(1-t)^{b-1} d t, a, b>0$. On the other hand, the fractal beta function (see [9]) is defined by

$$
B_{\alpha}(a, b)=\frac{1}{\Gamma(1+\alpha)} \int_{0}^{1} t^{\alpha(a-1)}(1-t)^{\alpha(b-1)}(d t)^{\alpha} .
$$


Utilizing the substitution $t=1 /(x+1)$, the above formula can be rewritten as

$$
B_{\alpha}(a, b)=\frac{1}{\Gamma(1+\alpha)} \int_{0}^{\infty} \frac{x^{\alpha(b-1)}}{\left(1^{\alpha}+x^{\alpha}\right)^{a+b}}(d x)^{\alpha},
$$

which will be a more suitable form for our further investigation.

\section{Main results}

In this section, we develop a unified treatment of fractal Hilbert-type inequalities. In other words, we will establish a pair of general Hilbert-type inequalities that covers particular fractal inequalities presented in the introduction.

Our main result refers to a general kernel which is local fractional continuous on the fractal surface $(a, b)^{2}:=(a, b) \times(a, b)$.

Theorem 1 Let $\frac{1}{p}+\frac{1}{q}=1, p>1,0<\alpha \leq 1$, and let $K \in C_{\alpha}(a, b)^{2}, \varphi, \psi \in C_{\alpha}(a, b)$ be nonnegative functions. If the functions $F$ and $G$ are defined by

$$
F^{p}(x)={ }_{a} I_{b}^{\alpha}\left(K(x, y) \psi^{-p}(y)\right), \quad G^{q}(y)={ }_{a} I_{b}^{\alpha}\left(K(x, y) \phi^{-q}(x)\right),
$$

then, for all non-negative functions $f, g \in C_{\alpha}(a, b)$, the inequalities

$$
{ }_{a} I_{b}^{\alpha}\left({ }_{a} I_{b}^{\alpha}(K(x, y) f(x) g(y))\right) \leq\left[{ }_{a} I_{b}^{\alpha}(\varphi F f)^{p}(x)\right]^{\frac{1}{p}}\left[{ }_{a} I_{b}^{\alpha}\left((\psi G g)^{q}(y)\right)\right]^{\frac{1}{q}}
$$

and

$$
{ }_{a} I_{b}^{\alpha}\left((G \psi)^{-p}(y)\left[{ }_{a} I_{b}^{\alpha}(K(x, y) f(x))\right]^{p}\right) \leq{ }_{a} I_{b}^{\alpha}(\varphi F f)^{p}(x)
$$

hold and are equivalent.

Proof The left-hand side of inequality (10) can be rewritten as

$$
\begin{aligned}
& \frac{1}{\Gamma^{2}(1+\alpha)} \int_{a}^{b} \int_{a}^{b} K(x, y) f(x) g(y)(d x)^{\alpha}(d y)^{\alpha} \\
& =\frac{1}{\Gamma^{2}(1+\alpha)} \int_{a}^{b} \int_{a}^{b} K(x, y) f(x) \frac{\varphi(x)}{\psi(y)} g(y) \frac{\psi(y)}{\varphi(x)}(d x)^{\alpha}(d y)^{\alpha},
\end{aligned}
$$

which is now suitable for the application of the Hölder inequality (7). Hence, we obtain

$$
\begin{aligned}
& \frac{1}{\Gamma^{2}(1+\alpha)} \int_{a}^{b} \int_{a}^{b} K(x, y) f(x) g(y)(d x)^{\alpha}(d y)^{\alpha} \\
& \leq\left[\frac{1}{\Gamma^{2}(1+\alpha)} \int_{a}^{b} \int_{a}^{b} K(x, y) f^{p}(x) \frac{\varphi^{p}(x)}{\psi^{p}(y)}(d x)^{\alpha}(d y)^{\alpha}\right]^{\frac{1}{p}} \\
& \quad \times\left[\frac{1}{\Gamma^{2}(1+\alpha)} \int_{a}^{b} \int_{a}^{b} K(x, y) g^{q}(y) \frac{\psi^{q}(y)}{\varphi^{q}(x)}(d x)^{\alpha}(d y)^{\alpha}\right]^{\frac{1}{q}} .
\end{aligned}
$$

Now, by virtue of the Fubini theorem (see, e.g., [14]), we can switch the order of integration in the double integral, so by taking into account the definitions of functions $F$ and $G$, we obtain (10), as claimed. 
Our next step is to show the equivalence of inequalities (10) and (11). Therefore, suppose that inequality (10) holds and define the function $g$ by

$$
g(y)=G^{-p}(y) \psi^{-p}(y)\left[\frac{1}{\Gamma(1+\alpha)} \int_{a}^{b} K(x, y) f(x)(d x)^{\alpha}\right]^{p-1}
$$

Now, since $\frac{1}{p}+\frac{1}{q}=1$, relation (10) implies the inequality

$$
\begin{aligned}
\frac{1}{\Gamma(1+\alpha)} \int_{a}^{b} G^{-p}(y) \psi^{-p}(y)\left[\frac{1}{\Gamma(1+\alpha)} \int_{a}^{b} K(x, y) f(x)(d x)^{\alpha}\right]^{p}(d y)^{\alpha} \\
=\frac{1}{\Gamma^{2}(1+\alpha)} \int_{a}^{b} \int_{a}^{b} K(x, y) f(x) g(y)(d x)^{\alpha}(d y)^{\alpha} \\
\leq\left[\frac{1}{\Gamma(1+\alpha)} \int_{a}^{b}(\varphi F f)^{p}(x)(d x)^{\alpha}\right]^{\frac{1}{p}}\left[\frac{1}{\Gamma(1+\alpha)} \int_{a}^{b}(\psi G g)^{q}(y)(d y)^{\alpha}\right]^{\frac{1}{q}} \\
=\left[\frac{1}{\Gamma(1+\alpha)} \int_{a}^{b}(\varphi F f)^{p}(x)(d x)^{\alpha}\right]^{\frac{1}{p}} \\
\quad \times\left[\frac{1}{\Gamma(1+\alpha)} \int_{a}^{b}(G \psi)^{q-p q}(y)\left[\frac{1}{\Gamma(1+\alpha)} \int_{a}^{b} K(x, y) f(x)(d x)^{\alpha}\right]^{q(p-1)}(d y)^{\alpha}\right]^{\frac{1}{q}},
\end{aligned}
$$

which reduces to (11).

On the other hand, suppose that inequality (11) holds. Then yet another application of the Hölder inequality yields

$$
\begin{aligned}
& \frac{1}{\Gamma^{2}(1+\alpha)} \int_{a}^{b} \int_{a}^{b} K(x, y) f(x) g(y)(d x)^{\alpha}(d y)^{\alpha} \\
&=\frac{1}{\Gamma(1+\alpha)} \int_{a}^{b}\left[\psi^{-1}(y) G^{-1}(y) \int_{a}^{b} K(x, y) f(x)(d x)^{\alpha}\right] \psi(y) G(y) g(y)(d y)^{\alpha} \\
& \leq {\left[\frac{1}{\Gamma(1+\alpha)} \int_{a}^{b} \psi^{-p}(y) G^{-p}(y)\left(\frac{1}{\Gamma(1+\alpha)} \int_{a}^{b} K(x, y) f(x)(d x)^{\alpha}\right)^{p}(d y)^{\alpha}\right]^{\frac{1}{p}} } \\
& \times\left[\frac{1}{\Gamma(1+\alpha)} \int_{a}^{b}(\psi G g)^{q}(y)(d y)^{\alpha}\right]^{\frac{1}{q}} \\
& \leq {\left[\frac{1}{\Gamma(1+\alpha)} \int_{a}^{b}(\varphi F f)^{p}(x)(d x)^{\alpha}\right]^{\frac{1}{p}}\left[\frac{1}{\Gamma(1+\alpha)} \int_{a}^{b}(\psi G g)^{q}(y)(d y)^{\alpha}\right]^{\frac{1}{q}}, }
\end{aligned}
$$

which provides (10). Consequently, inequalities (10) and (11) are equivalent.

It is not hard to see that our Theorem 1 covers fractal Hilbert-type inequalities (3) and (4) presented in the introduction. This follows by choosing a suitable power functions $\varphi$, $\psi$ appearing in relations (10) and (11). However, this will not be done at this moment. Namely, considering kernels $K_{1}(x, y)=1 / \max \left\{x^{\alpha}, y^{\alpha}\right\}$ and appearing in (3) and (4), we see that they possess a common property, they are both homogeneous functions. Therefore, our next step is to derive consequence of Theorem 1 which refers to homogeneous kernels. The fractal Hilbert-type inequalities presented in the introduction will then follow as simple consequences of our next result. 
Recall that the function $K \in C_{\alpha}(0, \infty)^{2}$ is said to be homogeneous of degree $-\alpha \lambda, \lambda>0$, if $K(t x, t y)=t^{-\alpha \lambda} K(x, y)$ for all $t>0$. In order to formulate and prove the corresponding result, we need the following definition. For a non-negative function $K \in C_{\alpha}(0, \infty)^{2}$, we define

$$
k_{\alpha}(\eta)={ }_{0} I_{\infty}^{\alpha} K(1, t) t^{-\alpha \eta}
$$

If nothing else is explicitly stated, we assume that the integral $k_{\alpha}(\eta)$ converges for considered values of $\eta$. Now, we are ready to establish a pair of fractal Hilbert-type inequalities that correspond to a class of homogeneous kernels.

Theorem 2 Let $\frac{1}{p}+\frac{1}{q}=1, p>1$, and let $f, g \in C_{\alpha}(0, \infty)$ be non-negative functions. If $K \in$ $C_{\alpha}(0, \infty)^{2}$ is a non-negative homogeneous function of degree $-\alpha \lambda, \lambda>0$, then the following inequalities hold:

$$
\begin{aligned}
& { }_{0} I_{\infty}^{\alpha}\left({ }_{0} I_{\infty}^{\alpha}(K(x, y) f(x) g(y))\right) \\
& \quad \leq L\left[{ }_{0} I_{\infty}^{\alpha}\left(x^{\alpha-\alpha \lambda+\alpha p\left(A_{1}-A_{2}\right)} f^{p}(x)\right)\right]^{\frac{1}{p}}\left[{ }_{0} I_{\infty}^{\alpha}\left(y^{\alpha-\alpha \lambda+\alpha q\left(A_{2}-A_{1}\right)} g^{q}(y)\right)\right]^{\frac{1}{q}}
\end{aligned}
$$

and

$$
\begin{aligned}
& { }_{0} I_{\infty}^{\alpha}\left[y^{\alpha(p-1)(\lambda-1)+\alpha p\left(A_{1}-A_{2}\right)}\left({ }_{0} I_{\infty}^{\alpha}(K(x, y) f(x))\right)^{p}\right] \\
& \quad \leq L^{p}\left[{ }_{0} I_{\infty}^{\alpha} x^{\alpha-\alpha \lambda+\alpha p\left(A_{1}-A_{2}\right)} f^{p}(x)\right]
\end{aligned}
$$

where $L=k_{\alpha}^{1 / p}\left(p A_{2}\right) k_{\alpha}^{1 / q}\left(2-\lambda-q A_{1}\right)$. In addition, relations (13) and (14) are equivalent.

Proof We employ inequalities (10) and (11) with power functions $\varphi(x)=x^{\alpha A_{1}}$ and $\psi(y)=$ $y^{\alpha A_{2}}$. Furthermore, making use of (9), it follows that

$$
F^{p}(x)=\frac{1}{\Gamma(1+\alpha)} \int_{0}^{\infty} K(x, y) y^{-\alpha p A_{2}}(d y)^{\alpha}
$$

and

$$
G^{q}(x)=\frac{1}{\Gamma(1+\alpha)} \int_{0}^{\infty} K(x, y) x^{-\alpha q A_{1}}(d x)^{\alpha} .
$$

In addition, since $K$ is a homogeneous function of degree $-\alpha \lambda, \lambda>0$, a change of variables $t=y / x$ provides

$$
\begin{aligned}
F^{p}(x) & =x^{\alpha-\alpha \lambda-\alpha p A_{2}} \frac{1}{\Gamma(1+\alpha)} \int_{0}^{\infty} K\left(1, \frac{y}{x}\right)\left(\frac{y}{x}\right)^{-\alpha p A_{2}} \frac{1}{x^{\alpha}}(d y)^{\alpha} \\
& =x^{\alpha-\alpha \lambda-\alpha p A_{2}} \frac{1}{\Gamma(1+\alpha)} \int_{0}^{\infty} K(1, t) t^{-\alpha p A_{2}}(d t)^{\alpha} \\
& =x^{\alpha-\alpha \lambda-\alpha p A_{2}} k_{\alpha}\left(p A_{2}\right)
\end{aligned}
$$

due to (6). Following the lines as in the previous step, we also obtain

$$
G^{q}(y)=y^{\alpha-\alpha \lambda-\alpha q A_{1}} \frac{1}{\Gamma(1+\alpha)} \int_{0}^{\infty} K(t, 1) t^{-\alpha q A_{1}}(d t)^{\alpha} .
$$


Now, yet another application of the change of variables rule (6) with $u=t^{-1}$, gives

$$
\begin{aligned}
G^{q}(y) & =-y^{\alpha-\alpha \lambda-\alpha q A_{1}} \frac{1}{\Gamma(1+\alpha)} \int_{0}^{\infty} K\left(1, \frac{1}{t}\right)\left(\frac{1}{t}\right)^{\alpha \lambda+\alpha q A_{1}-2 \alpha}\left(-t^{-2 \alpha}\right)(d t)^{\alpha} \\
& =y^{\alpha-\alpha \lambda-\alpha q A_{1}} \frac{1}{\Gamma(1+\alpha)} \int_{0}^{\infty} K(1, u) u^{\alpha \lambda+\alpha q A_{1}-2 \alpha}(d u)^{\alpha} \\
& =y^{\alpha-\alpha \lambda-\alpha q A_{1}} k_{\alpha}\left(2-\lambda-q A_{1}\right) .
\end{aligned}
$$

Finally, inequalities (13) and (14) follow from relations (10), (11), (15), and (16).

It should be noticed here that Theorem 2 holds for arbitrary parameters $A_{1}$ and $A_{2}$ such that the constant $L$ and the integrals on the right-hand sides of (13) and (14) are convergent.

Generally speaking, we are not able to prove whether or not the constants $L$ and $L^{p}$ appearing on the right-hand sides of (13) and (14) are the best possible. However, it turns out that these constants are the best possible for a wide set of parameters $A_{1}, A_{2}$ and a weak condition on the kernel $K$. In order to establish the corresponding result, we first need the following lemma.

Lemma 1 Let $\lambda>0$, and let $\frac{1}{p}+\frac{1}{q}=1, p>1$. If $K \in C_{\alpha}(0, \infty)^{2}$ is a non-negative function such that $K(1, t)$ is bounded on $(0,1)$, then the following relation holds:

$$
{ }_{1} I_{\infty}^{\alpha}\left[x^{-\alpha(1+\varepsilon)}{ }_{0}^{\alpha} I_{1 / x}^{\alpha}\left(t^{-\alpha p A_{2}-\frac{\varepsilon \alpha}{q}} K(1, t)\right)\right] \leq O(1), \quad \varepsilon \rightarrow 0^{+}
$$

where $A_{2} \leq \frac{1}{2 p}$.

Proof From the hypotheses, we have $K(1, t) \leq C$ for some $C>0$ and every $t \in(0,1)$. Then it follows that

$$
\begin{aligned}
{ }_{1} I_{\infty}^{\alpha} & {\left[x^{-\alpha(1+\varepsilon)}{ }_{0}^{\alpha} I_{1 / x}^{\alpha}\left(t^{-\alpha p A_{2}-\frac{\varepsilon \alpha}{q}} K(1, t)\right)\right] } \\
& \leq C_{1} I_{\infty}^{\alpha}\left[x^{-\alpha}{ }_{0} I_{1 / x}^{\alpha}\left(t^{-\frac{\alpha}{2}-\frac{\varepsilon \alpha}{q}}\right)\right] .
\end{aligned}
$$

Furthermore, utilizing the change of variables rule (6) with $g(t)=t^{\frac{1}{2}-\frac{\varepsilon}{q}},\left[g^{\prime}(t)\right]^{\alpha}=\left(\frac{1}{2}-\right.$ $\left.\frac{\varepsilon}{q}\right)^{\alpha} t^{-\frac{\alpha}{2}-\frac{\varepsilon \alpha}{q}}$, we obtain

$$
\begin{aligned}
{ }_{1} I_{\infty}^{\alpha} & {\left[x^{-\alpha}\left({ }_{0} I_{1 / x}^{\alpha}\left(t^{-\frac{\alpha}{2}-\frac{\varepsilon \alpha}{q}}\right)\right)\right] } \\
& =\frac{1}{\Gamma^{2}(1+\alpha)} \int_{1}^{\infty} x^{-\alpha}\left(\int_{0}^{1 / x} t^{-\frac{\alpha}{2}-\frac{\varepsilon \alpha}{q}}(d t)^{\alpha}\right)(d x)^{\alpha} \\
& =\frac{1}{\Gamma^{2}(1+\alpha)} \int_{1}^{\infty} x^{-\alpha}\left(\frac{1}{\left(\frac{1}{2}-\frac{\varepsilon}{q}\right)^{\alpha}} \int_{0}^{x^{-\frac{1}{2}+\frac{\varepsilon}{q}}}(d u)^{\alpha}\right)(d x)^{\alpha} \\
& =\frac{1}{\Gamma^{2}(1+\alpha)\left(\frac{1}{2}-\frac{\varepsilon}{q}\right)^{\alpha}} \int_{1}^{\infty} x^{-\frac{3 \alpha}{2}+\frac{\varepsilon \alpha}{q}}(d x)^{\alpha} .
\end{aligned}
$$


Now, if $g(x)=x^{-\frac{1}{2}+\frac{\varepsilon}{q}}$, then $\left[g^{\prime}(x)\right]^{\alpha}=-\left(\frac{1}{2}-\frac{\varepsilon}{q}\right)^{-\alpha} x^{-\frac{3 \alpha}{2}+\frac{\varepsilon \alpha}{q}}$, so by (6), we have

$$
{ }_{1} I_{\infty}^{\alpha}\left[x^{-\alpha}{ }_{0} I_{1 / x}^{\alpha}\left(t^{-\frac{\alpha}{2}-\frac{\varepsilon \alpha}{q}}\right)\right]=\frac{1}{\Gamma^{2}(1+\alpha)\left(\frac{1}{2}-\frac{\varepsilon}{q}\right)^{2 \alpha}} .
$$

Finally, combining (18) and (19), we obtain (17), as claimed.

Our next intention is to impose the condition on parameters $A_{1}$ and $A_{2}$ for which the constants appearing on the right-hand sides of inequalities (13) and (14) are the best possible. It should be noticed here that if

$$
p A_{2}+q A_{1}=2-\lambda
$$

then the constant $L$ from Theorem 2 reduces to the form without exponents, i.e.,

$$
L^{*}=k_{\alpha}\left(p A_{2}\right)
$$

We will show that if the parameters $A_{1}$ and $A_{2}$ are related by (20), then the constants appearing on the right-hand sides of (13) and (14) are the best possible. In fact, if (20) holds, inequalities (13) and (14) reduce to

$$
\begin{aligned}
& { }_{0} I_{\infty}^{\alpha}\left({ }_{0} I_{\infty}^{\alpha}(K(x, y) f(x) g(y))\right) \\
& \quad \leq L^{*}\left[{ }_{0} I_{\infty}^{\alpha}\left(x^{\alpha p q A_{1}-\alpha} f^{p}(x)\right)\right]^{\frac{1}{p}}\left[{ }_{0} I_{\infty}^{\alpha}\left(y^{\alpha p q A_{2}-\alpha} g^{q}(y)\right)\right]^{\frac{1}{q}}
\end{aligned}
$$

and

$$
\begin{gathered}
{ }_{0} I_{\infty}^{\alpha}\left[y^{\alpha p(\lambda-1)+\alpha p q A_{1}}\left({ }_{0} I_{\infty}^{\alpha}(K(x, y) f(x))\right)^{p}\right] \\
\leq\left(L^{*}\right)^{p}\left[{ }_{0} I_{\infty}^{\alpha}\left(x^{\alpha p q A_{1}-\alpha} f^{p}(x)\right)\right]
\end{gathered}
$$

where $L^{*}$ is defined by (21).

Theorem 3 Suppose that $\frac{1}{p}+\frac{1}{q}=1, p>1$, and let $f, g \in C_{\alpha}(0, \infty)$ be non-negative functions. Further, let $K \in C_{\alpha}(0, \infty)^{2}$ be a non-negative homogeneous function of degree $-\alpha \lambda, \lambda>0$, such that $K(1, t)$ is bounded on $(0,1)$. If the parameters $A_{1}$ and $A_{2}$ satisfy relation $p A_{2}+$ $q A_{1}=2-\lambda$, then the constants $L^{*}$ and $\left(L^{*}\right)^{p}$ appearing on the right-hand sides of $(22)$ and (23) are the best possible.

Proof Let $f(x)=x^{-\alpha q A_{1}-\frac{\varepsilon \alpha}{p}} \chi_{[1, \infty)}(x)$ and $g(y)=y^{-\alpha p A_{2}-\frac{\varepsilon \alpha}{q}} \chi_{[1, \infty)}(y)$, where $\chi_{A}$ stands for a characteristic function of a set $A$. Now, let us suppose that there exists a smaller constant $0<M<L^{*}$ such that inequality (22) holds. Denote by $J$ the right-hand side of inequality (22). Then, with the above defined functions $f$ and $g$, we have

$$
\begin{aligned}
J & =M\left(\frac{1}{\Gamma(1+\alpha)} \int_{1}^{\infty} x^{-\alpha \varepsilon-\alpha}(d x)^{\alpha}\right)^{\frac{1}{p}}\left(\frac{1}{\Gamma(1+\alpha)} \int_{1}^{\infty} y^{-\alpha \varepsilon-\alpha}(d y)^{\alpha}\right)^{\frac{1}{q}} \\
& =\frac{M}{\varepsilon^{\alpha} \Gamma(1+\alpha)}
\end{aligned}
$$


Further, utilizing substitution $t=\frac{y}{x}$ and taking into account Lemma 1 , we obtain the following estimate:

$$
\begin{aligned}
{ }_{0} I_{\infty}^{\alpha} & \left({ }_{0} I_{\infty}^{\alpha}(K(x, y) f(x) g(y))\right) \\
& ={ }_{1} I_{\infty}^{\alpha}\left[x^{-\alpha q A_{1}-\frac{\alpha \varepsilon}{p}} I_{\infty}^{\alpha}\left(y^{-\alpha p A_{2}-\frac{\alpha \varepsilon}{q}} K(x, y)\right)\right] \\
& ={ }_{1} I_{\infty}^{\alpha}\left[x^{-\alpha(1+\varepsilon)}\left({ }_{0} I_{\infty}^{\alpha}\left(t^{-\alpha p A_{2}-\frac{\alpha \varepsilon}{q}} K(1, t)\right)-{ }_{0} I_{1 / x}^{\alpha}\left(t^{-\alpha p A_{2}-\frac{\alpha \varepsilon}{q}} K(1, t)\right)\right)\right] \\
& \geq \frac{1}{\varepsilon^{\alpha} \Gamma(1+\alpha)}\left(k_{\alpha}\left(p A_{2}+\frac{\varepsilon}{q}\right)+o(1)\right) .
\end{aligned}
$$

Moreover, from (22), (24), and (25), we get

$$
k_{\alpha}\left(p A_{2}+\frac{\varepsilon}{q}\right)+o(1) \leq M
$$

Now, by letting $\varepsilon \rightarrow 0^{+}$, it follows that relation (26) contradicts with our assumption $M<$ $L^{*}=k_{\alpha}\left(p A_{2}\right)$.

Finally, equivalence of inequalities (22) and (23) means that the constant $\left(L^{*}\right)^{p}=$ $\left[k_{\alpha}\left(p A_{2}\right)\right]^{p}$ is also the best possible in (23). The proof is now completed.

\section{Applications}

In this section, we apply our Theorems 2 and 3 to some particular settings. More precisely, we will consider derived fractal Hilbert-type inequalities for some particular choices of homogeneous kernels and parameters $A_{1}$ and $A_{2}$ related by (20).

Our first example refers to the kernel $K_{1}(x, y)=1 / \max \left\{x^{\alpha \lambda}, y^{\alpha \lambda}\right\}, \lambda>0$, and the parameters $A_{1}=\frac{2-\lambda}{2 q}, A_{2}=\frac{2-\lambda}{2 p}$. Obviously, $K_{1}$ is a homogeneous function of degree $-\alpha \lambda$ and $K_{1}(1, t)$ is bounded on $(0,1)$. Moreover, the parameters $A_{1}$ and $A_{2}$ satisfy condition (20), so the hypotheses of Theorem 3 are satisfied. In addition, the constant in inequality (22), denoted here by $L_{1}^{*}$, reduces to

$$
L_{1}^{*}=k_{\alpha}\left(1-\frac{\lambda}{2}\right)=\frac{2^{\alpha+1}}{\lambda^{\alpha} \Gamma(1+\alpha)} .
$$

This follows by virtue of (5), after a straightforward calculation. The corresponding result covers fractal Hilbert-type inequalities presented in the introduction.

Corollary 1 Let $\frac{1}{p}+\frac{1}{q}=1, p>1, \lambda>0$, and let $f, g \in C_{\alpha}(0, \infty)$ be non-negative functions. Then the inequalities

$$
\begin{aligned}
& { }_{0} I_{\infty}^{\alpha}\left({ }_{0} I_{\infty}^{\alpha}\left(\frac{f(x) g(y)}{\max \left\{x^{\alpha \lambda}, y^{\alpha \lambda}\right\}}\right)\right) \\
& \quad \leq \frac{2^{\alpha+1}}{\lambda^{\alpha} \Gamma(1+\alpha)}\left[{ }_{0} I_{\infty}^{\alpha}\left(x^{\alpha(p-1)-\frac{\alpha \lambda p}{2}} f^{p}(x)\right)\right]^{\frac{1}{p}}\left[{ }_{0} I_{\infty}^{\alpha}\left(y^{\alpha(q-1)-\frac{\alpha \lambda q}{2}} g^{q}(y)\right)\right]^{\frac{1}{q}}
\end{aligned}
$$

and

$$
{ }_{0} I_{\infty}^{\alpha}\left(y^{\frac{\alpha p \lambda}{2}}\left[{ }_{0} I_{\infty}^{\alpha}\left(\frac{f(x)}{\max \left\{x^{\alpha \lambda}, y^{\alpha \lambda}\right\}}\right)\right]^{p}\right)
$$




$$
\leq\left(\frac{2^{\alpha+1}}{\lambda^{\alpha} \Gamma(1+\alpha)}\right)^{p}\left[{ }_{0} I_{\infty}^{\alpha}\left(x^{\alpha(p-1)-\frac{\alpha \lambda p}{2}} f^{p}(x)\right)\right]
$$

hold and the constants appearing on their right-hand sides are the best possible.

Remark 1 It should be noticed here that our Corollary 1 is an extension of fractal Hilberttype inequalities discussed in the introduction. More precisely, by substituting $\lambda=1$ in inequalities (27) and (28), we obtain relations (3) and (4).

Our next example deals with a homogeneous kernel $K_{2}(x, y)=1 /\left(x^{\alpha}+y^{\alpha}\right)^{\lambda}, \lambda>0$, and the parameters $A_{1}=\frac{2-\lambda}{2 q}, A_{2}=\frac{2-\lambda}{2 p}$. Clearly, $K_{2}$ is a homogeneous function of degree $-\alpha \lambda$ and $K_{2}(1, t)$ is bounded on $(0,1)$. Now, if the parameters $A_{1}$ and $A_{2}$ are related by (20), then the constant in inequality (22), denoted here by $L_{2}^{*}$, can be expressed in terms of the fractal beta function $B_{\alpha}$. More precisely, utilizing the integral representation (8), it follows that

$$
L_{2}^{*}=B_{\alpha}\left(\frac{\lambda}{2}, \frac{\lambda}{2}\right)
$$

so we have the following consequence.

Corollary 2 Let $\frac{1}{p}+\frac{1}{q}=1, p>1$, and let $f, g \in C_{\alpha}(0, \infty)$ be non-negative functions. Then the inequalities

$$
\begin{aligned}
& { }_{0} I_{\infty}^{\alpha}\left({ }_{0} I_{\infty}^{\alpha}\left(\frac{f(x) g(y)}{\left(x^{\alpha}+y^{\alpha}\right)^{\lambda}}\right)\right) \\
& \quad \leq B_{\alpha}\left(\frac{\lambda}{2}, \frac{\lambda}{2}\right)\left[{ }_{0} I_{\infty}^{\alpha}\left(x^{\alpha(p-1)-\frac{\alpha \lambda p}{2}} f^{p}(x)\right)\right]^{\frac{1}{p}}\left[{ }_{0} I_{\infty}^{\alpha}\left(y^{\alpha(q-1)-\frac{\alpha \lambda q}{2}} g^{q}(y)\right)\right]^{\frac{1}{q}}
\end{aligned}
$$

and

$$
\begin{aligned}
& { }_{0} I_{\infty}^{\alpha}\left(y^{\frac{\alpha p \lambda}{2}}\left[{ }_{0} I_{\infty}^{\alpha}\left(\frac{f(x)}{\left(x^{\alpha}+y^{\alpha}\right)^{\lambda}}\right)\right]^{p}\right) \\
& \quad \leq\left(B_{\alpha}\left(\frac{\lambda}{2}, \frac{\lambda}{2}\right)\right)^{p}\left[{ }_{0} I_{\infty}^{\alpha}\left(x^{\alpha(p-1)-\frac{\alpha \lambda p}{2}} f^{p}(x)\right)\right]
\end{aligned}
$$

hold and the constants appearing on their right-hand sides are the best possible.

At the end of the paper, we discuss the case of the kernel $K_{3}(x, y)=1 /\left(x^{\alpha}+y^{\alpha}\right)$. In this setting, the constant in inequality (22), denoted here by $L_{3}^{*}$, becomes

$$
L_{3}^{*}=B_{\alpha}\left(p A_{2}, 1-p A_{2}\right)
$$

provided that $p A_{2}+q A_{1}=1$. The resulting pair of relations is given in the following result.

Corollary 3 Let $\frac{1}{p}+\frac{1}{q}=1, p>1$, and let $f, g \in C_{\alpha}(0, \infty)$ be non-negative functions. If $A_{1}$ and $A_{2}$ are real parameters such that $p A_{2}+q A_{1}=2 \alpha-1$, then the inequalities

$$
\begin{aligned}
& { }_{0} I_{\infty}^{\alpha}\left({ }_{0} I_{\infty}^{\alpha}\left(\frac{f(x) g(y)}{x^{\alpha}+y^{\alpha}}\right)\right) \\
& \quad \leq B_{\alpha}\left(p A_{2}, 1-p A_{2}\right)\left[{ }_{0} I_{\infty}^{\alpha}\left(x^{\alpha p q A_{1}-\alpha} f^{p}(x)\right)\right]^{\frac{1}{p}}\left[{ }_{0} I_{\infty}^{\alpha}\left(y^{\alpha p q A_{2}-\alpha} g^{q}(y)\right)\right]^{\frac{1}{q}}
\end{aligned}
$$


and

$$
\begin{aligned}
& { }_{0} I_{\infty}^{\alpha}\left(y^{\alpha p q A_{1}-\alpha}\left[{ }_{0} I_{\infty}^{\alpha}\left(\frac{f(x)}{x^{\alpha}+y^{\alpha}}\right)\right]^{p}\right) \\
& \quad \leq\left(B_{\alpha}\left(p A_{2}, 1-p A_{2}\right)\right)^{p}\left[{ }_{0} I_{\infty}^{\alpha}\left(x^{\alpha p q A_{1}-\alpha} f^{p}(x)\right)\right]
\end{aligned}
$$

hold and the constants appearing on their right-hand sides are the best possible.

Remark 2 In particular, if $A_{1}=A_{2}=\frac{1}{p q}$, then the constant $L_{2}^{*}$ from Corollary 2 reduces to the form $L_{2}^{*}=B_{\alpha}\left(\frac{1}{q}, \frac{1}{p}\right)$. In this case, the weight functions $x \rightarrow x^{\alpha p q A_{1}-\alpha}$ and $y \rightarrow y^{\alpha p q A_{2}-\alpha}$ in inequalities (29) and (30) disappear. The resulting pair of non-weighted inequalities is an immediate fractal extension of the initial Hilbert-type inequalities (1) and (2). It should be noticed here that if $\alpha=1$, then $B_{\alpha}\left(\frac{1}{q}, \frac{1}{p}\right)=B\left(\frac{1}{q}, \frac{1}{p}\right)=\frac{\pi}{\sin \frac{\pi}{p}}$.

\section{Conclusion}

In the present study, we have established a unified treatment of fractal Hilbert-type inequalities. First, we have derived a pair of equivalent Hilbert-type inequalities with a general kernel and weight functions. A particular emphasis has been devoted to a class of homogeneous kernels. In addition, we have established conditions under which the constants appearing in the corresponding inequalities are the best possible. As an application, our results have been compared with some previously known ones from the literature.

Funding

The first author would like to thank the Asia Research Center at the National University of Mongolia and the Korea Foundation of Advanced Studies for supporting this research (Project No. P2017-2479, 2018).

\section{Competing interests}

The authors declare that they have no competing interests.

Authors' contributions

All authors read and approved the final version of the manuscript.

\section{Author details}

${ }^{1}$ Department of Mathematics, National University of Mongolia, Ulaanbaatar, Mongolia. ${ }^{2}$ Faculty of Electrical Engineering and Computing, University of Zagreb, Zagreb, Croatia. ${ }^{3}$ Faculty of Teacher Education, University of Zagreb, Zagreb, Croatia.

\section{Publisher's Note}

Springer Nature remains neutral with regard to jurisdictional claims in published maps and institutional affiliations.

Received: 30 August 2018 Accepted: 17 April 2019 Published online: 30 April 2019

\section{References}

1. Abdeldaim, A., El-Deeb, A.A.: On generalized of certain retarded nonlinear integral inequalities and its applications in retarded integro-differential equations. Appl. Math. Comput. 256, 375-380 (2015)

2. Adiyasuren, V., Batbold, T., Krnić, M.: Multiple Hilbert-type inequalities involving some differential operators. Banach J. Math. Anal. 10(2), 320-337 (2016)

3. Batbold, T., Krnić, M., Pečarić, J., Vuković, P.: Further Development of Hilbert-Type Inequalities. Element, Zagreb (2017)

4. Budak, H., Sarikaya, M.Z., Yildirim, H.: New inequalities for local fractional integrals. Iran. J. Sci. Technol., Trans. A, Sci. 41(4), 1039-1046 (2017)

5. Chen, G.-S.: Generalizations of Hölder's and some related integral inequalities on fractal space. J. Funct. Spaces Appl. 2013, Article ID 198405 (2013)

6. Chen, G.-S., Srivastava, J.M., Wang, P., Wei, W.: Some further generalizations of Hölder's inequality and related results on fractal space. Abstr. Appl. Anal. 2014, Article ID 832802 (2014)

7. El-Deeb, A.A., Cheung, W.-S.: Some reverse Hölder inequalities with Specht's ratio on time scales. J. Nonlinear Sci. Appl. 11, 444-455 (2018)

8. Hardy, G.H., Littlewood, J.E., Pólya, G.: Inequalities, 2nd edn. Cambridge University Press, Cambridge (1967) 
9. Jumarie, G.: Fractional Euler's integral of first and second kinds. Application to fractional Hermite's polynomials and to probability density of fractional order. J. Appl. Math. Inform. 28(1-2), 257-273 (2010)

10. Krnić, M., Pečarić, J., Perić, I., Vuković, P.: Recent Advances in Hilbert-Type Inequalities. Element, Zagreb (2012)

11. Liu, Q.: A Hilbert-type fractional integral inequality with the kernel of Mittag-Leffler function and its applications. Math. Inequal. Appl. 21(3), 729-737 (2018)

12. Liu, Q., Chen, D.: A Hilbert-type integral inequality on the fractal spaces. Integral Transforms Spec. Funct. 28(10), 772-780 (2017)

13. Liu, Q., Sun, W.: A Hilbert-type fractal integral inequality and its applications. J. Inequal. Appl. 2017, 83 (2017)

14. Rudin, W.: Real and Complex Analysis, 3rd edn. McGraw-Hill, New York (1987)

15. Saker, S.H., El-Deeb, A.A., Rezk, H.M., Agarwal, R.P.: On Hilbert's inequalities on time scales. Appl. Anal. Discrete Math. 11, 399-423 (2017)

16. Sarikaya, M.Z., Budak, H.: Generalized Ostrowski type inequalities for local fractional integrals. Proc. Am. Math. Soc. 145(4), 1527-1538 (2017)

17. Sarikaya, M.Z., Tunc, T., Budak, H.: On generalized some integral inequalities for local fractional integrals. Appl. Math. Comput. 276, 316-323 (2016)

18. Yang, X.J.: Local Fractional Functional Analysis and Its Applications. Asian Academic Publisher Limited, Hong Kong (2011)

19. Yang, X.J.: Advanced Local Fractional Calculus and Its Applications. Word Science Publishers, New York (2012)

\section{Submit your manuscript to a SpringerOpen ${ }^{\circ}$ journal and benefit from:}

- Convenient online submission

- Rigorous peer review

- Open access: articles freely available online

- High visibility within the field

- Retaining the copyright to your article

Submit your next manuscript at $\gg$ springeropen.com 